\title{
Light Relations in Plant Canopies
}

\author{
S. B. Idso and C. T. de Wit
}

\begin{abstract}
A theory of light relations in plant canopies is presented which has potential applications in remote sensing and photosynthetic modeling of plant canopies. Predictions of the model are compared with field measurements of light reflection and transmission in a corn crop. Both reflection at the top of the canopy and transmission at the bottom are predicted within $1 \%$ of the measured values. Profiles connecting these upper and lower limits are equally well approximated. Variations in the predictions with altitude angle of the sun are confirmed by the observation of several investigators.
\end{abstract}

\section{Introduction}

The interaction of direct solar radiation and diffuse skylight with the leaves of a plant canopy is a phenomenon which requires detailed understanding before proper analyses can be made of remotely acquired electrooptical data obtained by aircraft or spacecraft passing over cropped surfaces. Early theoretical studies of this problem were based on a one-parameter relation equivalent to the Bouguert-Lambert law; ${ }^{1}$ these works have been reviewed briefly by Myers and Allen. ${ }^{2}$ A recent advancement has been the application of the Kubelka and $\mathrm{Munk}^{3}$ (K-M) two-parameter theory to the interaction of light with a plant community by Allen and Richardson. ${ }^{4}$ Whereas the Bouguer-Lambert law makes no prediction regarding the reflectance of a medium, Allen and Richardson were able to use $K-M$ theory to predict accurately both reflectance and transmittance from two, four, six, and eight stacked cotton leaves in a spectrophotometer. They indicated also that their procedures were sufficiently general to apply to an actual plant canopy, although this application was not made.

In studying plant canopies, however, it becomes apparent that the extension of $\mathrm{K}-\mathrm{MI}$ theory proposed by Allen and Richardson is not precisely valid, the reason being that $\mathrm{K}-\mathrm{M}$ theory requires a uniform distribution of leaf inclinations. Nichiporovich ${ }^{5}$ and de Wit $^{6}$ both have experimental evidence indicating that probably no plant canopy has leaves distributed

S. B. Idso is with the U.S. Water Conservation Laboratory, Phoenix, Arizona 85040; C. T. de Wit is with the Agricultural University, Wageningen, The Netherlands.

Received 23 June 1969. in such a manner. Thus, in a real canopy, the $\mathrm{K}-\mathbf{M}$ scattering and absorption coefficients would be variables dependent upon the altitude angle of the.sun. Or, viewing the situation from a different perspective, the K-M extension proposed by Allen and Richardson would predict a canopy reflectance that would not vary over the day. It is well known, however, that re flectance from a cropped surface usually exhibits. a diurnal variation. This effect has been observed with alfalfa, barley, wheat, oats, cotton, and sorghum by Fritschen, ${ }^{7}$ with plant cane and pangola grass by Chia, ${ }^{8}$ with short grass and kale by Monteith and Szeicz, ${ }^{9}$ and with sod by Idso et al. ${ }^{10}$ to name a few specific examples. In these studies, reflectance was found to increase by $4-6 \%$ at low solar elevations, compared to values obtained at solar noon.

It is the purpose of this paper to describe a theory of light relations in plant canopies which overcomes the limitations of IK-M theory. The new theory will be applied to an actual crop growing in a field situation, and its prediction of crop reflectance and transmittance plus vertical profiles of these two parameters will be compared with the results of field measurements.

\section{Theory}

Since the distribution of leaf inclinations in a plant canopy will influence the light relations therein, it is necessary to somehow characterize this factor. A useful convention for this representation is the leaf distribution function, introduced by de Wit. ${ }^{6}$. It is defined as the frequency distribution of leaf inclinations from the horizontal. Figure 1 depicts six examples: planophile canopies, where horizontal leaves are most frequent; erectophile canopies, where vertical leaves are most frequent; plagiophile canopies, where leaves at some oblique inclination are most frequent; $e x-$ tremophile canopies, where leaves are least frequent at oblique inclinations; spherical canopies, where the relative frequency of leaf inclinations is the same as the 
$A$
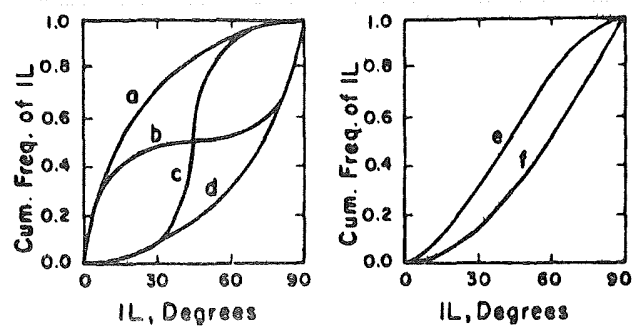

Fig. 1. (a) The four general types of leaf distribution functions: a. planophile; b. extremophile; c. plagiophile; d. erectophile. (b) Two special lesf distribution functions: e. com canopy (measured); 1. spherical.

relative frequency of the inclinations of the surface elements of a sphere; and a measured leaf distribution function for corn.

The first step of what we may call the de Wit-Idso (D-I) theory is to derive what de Wit ${ }^{6}$ termed the light distribution function of a crop from its leaf distribution function. Since the incident light on a leaf due to direct light is proportional to the sine of the angle $(L S)$ between the leaf and the rays of the sun, this light distribution function is defined as the cumulative frequency distribution of intercepted light as a function of $\sin (L S)$.

In obtaining the light distribution function of a particular crop, it is first necessary to calculate for nine different altitude angles of the sun $\left(I S=5^{\circ}, 15^{\circ}, \ldots\right.$, $85^{\circ}$ ) and nine different leaf inclinations ( $I L=5^{\circ}$, $15^{\circ}, \ldots, 85^{\circ}$ ) the probabilities of having a light ray intercepted by a leaf with the sine of its angle to the light equal to or smaller than $\sin (L S)$. The equations for accomplishing this feat were first derived by de Wit. ${ }^{6}$ Since his development was very abbreviated, a more thorough derivation has been made and is included in Appendix A of this paper. Since the eightyone sets of calculations which must be made for all pairs $(I S, I L)$ are quite tedious, this work is done by computer. Since they need only be done once, however, and the answers are identically useful for all problems of this type, results are included for the benefit of other investigators in Appendix B.

The calculation of a crop light distribution function from the data in Appendix B and the crop's leaf dis- tribution function now proceeds on the basis that the light intercepted by leaves in any leaf inclination class is proportional to the number of leaves in this class and to the projected area in the direction of the sun of one unit leaf area of the class. Thus, by computer, nine integrated crop light distribution functions for sun altitudes of $5^{\circ}, 15^{\circ}, \ldots, 85^{\circ}$ are obtained. The results for a corn crop are contained in Table I. For purposes which will be apparent later, it is also desirable to transform these fractions of intercepted light into fractions of leaves which receive the light within the various $\sin (L S)$ intervals. The formula which accomplishes this transformation is

$$
F R(J)=R(J) / S R
$$

where

$$
\begin{gathered}
R(J)=[S(J)-S(J-1)] / S N(J), \\
S R=\sum_{J=1}^{10} R(J),
\end{gathered}
$$

and $S(J)=$ cumulative frequency (fraction) of intercepted light, with $J=1,2, \ldots, 10$ corresponding to light intercepted at values of $\sin (L S) \leq 0.1,0.2$, $\ldots, 1.0 . \quad S N(J)=0.05,0.15, \ldots, 0.95$, corresponding to $J=1,2, \ldots, 10$. Results of these calculations for a corn crop are contained in Table II.

Up to this point, D-I theory has been concerned with what in essence is the uppermost layer of leaves in the plant canopy; and the next step is to consider the extinction of the solar beam as it penetrates the canopy foliage. In this context, leaf area index $(L A I)$ and canopy density $(S)$ are introduced. The latter of these two parameters was first used by Monsi and Saeki ${ }^{11}$ to describe the penetration of light into canopies of perfectly absorbing horizontal leaves. De Wit, ${ }^{6}$ however, generalized it to the case of distributively inclined leaves that do reflect and transmit light. Its meaning then became somewhat more vague. Nevertheless, it may be considered to be characteristic of the extent to which the leaves are uniformly distributed in inclination; a random distribution having $S=0$, and $S=1$ implying all leaves positioned horizontally. A good value for corn is 0.1 .

Considering first direct light incident at an inclination IS, the interception of this light is proportional to the projection of one unit leaf area in the direction of the

Table 1. The Light Distribution Functions for a Corn Crop for Sun Inclinations $15=5^{\circ}, 15^{\circ}, \ldots, \ldots 5^{\circ}$

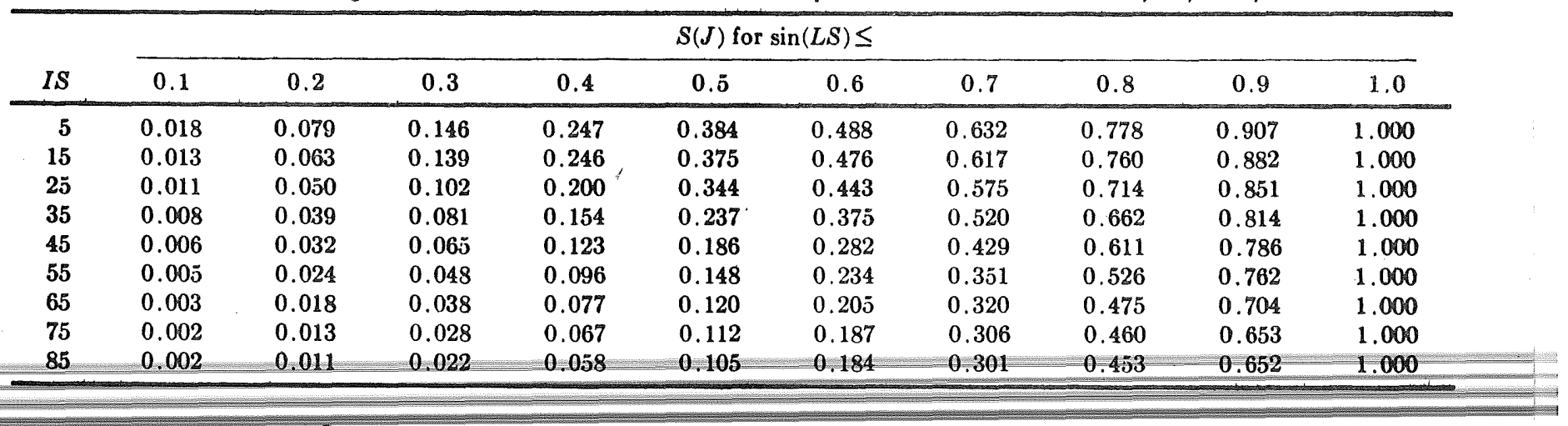


Table 11. The Fractions of Sunlit Leaves of a Corn Crop Receiving Direct Light within Various sin(LS) Intervals for Sur Inclinations $I S=5^{\circ}, 15^{\circ}, \ldots, 85^{\circ}$

\begin{tabular}{|c|c|c|c|c|c|c|c|c|c|c|}
\hline \multicolumn{11}{|c|}{$F R(J)$ for $\sin (L S) \leq$} \\
\hline$I S$ & 0.1 & 0.2 & 0.3 & 0.4 & 0.5 & 0.6 & 0.7 & 0.8 & 0.9 & 1.0 \\
\hline 5 & 0.145 & 0.164 & 0.108 & 0.116 & 0.123 & 0.076 & 0.089 & 0.078 & 0.061 & 0.039 \\
\hline 15 & 0.111 & 0.142 & 0.129 & 0.130 & 0.122 & 0.078 & 0.092 & 0.081 & 0.061 & 0.053 \\
\hline 25 & (). 101 & 0.120 & 0.096 & 0.129 & 0.147 & 0.083 & 0.093 & 0.085 & 0.074 & 0.072 \\
\hline 35 & 0.081 & (1. 105 & 0.085 & 0.106 & 0.094 & 0.128 & 0.113 & 0.096 & 0.091 & 0.100 \\
\hline $4 \tilde{3}$ & 0.066 & 0.096 & 0.073 & 0.092 & 0.078 & 0.097 & 0.125 & 0.134 & 0.114 & 0.125 \\
\hline $5 \bar{j}$ & 0.060 & 0.076 & 0.057 & 0.082 & 0.069 & 0.093 & 0.108 & 0.139 & 0.166 & 0.150 \\
\hline 65 & 0.038 & 0.064 & 0.051 & 0.071 & 0.061 & 0.099 & 0.113 & 0.132 & 0.172 & 0.199 \\
\hline 75 & 0.027 & 0.049 & 0.040 & 0.074 & 0.067 & 0.091 & 0.122 & 0.137 & 0.151 & 0.243 \\
\hline 85 & 0.027 & 0.041 & 0.030 & 0.070 & 0.071 & 0.097 & 0.122 & 0.137 & 0.158 & 0.248 \\
\hline
\end{tabular}

light, $O P(I S)$, averaged for the crop as a whole, and inversely proportional to the projection of one unit soil area in the same direction. Since the latter projection is equal to the sine of $I S$, the direct light that penetrates the canopy without interception may be described by the relation

$$
I=I O\left[1-S \frac{O P(I S)}{\sin (I S)}\right]^{L A I / S}
$$

where $I O$ is the amount of light arriving from the vertical direction at the canopy and $L A I / S$ is the number of layers defined for the crop foliage, e.g., for $L A I=3.5$ (in a case to be considered shortly) and $S=0.1$, the crop is considered to be composed of thirty-five vertically stacked layers.

If we let $K$ represent the nine altitudinal positions of the sun and $N$ represent the number of canopy layers, we may calculate the set of numbers

$$
X(K, N)=\left[1-s \frac{O P(I S)}{\sin (I S)}\right]^{N} .
$$

Since Eq. (4) describes the penetration of direct light into the canopy, these $X(K, N)$ values thus represent the fractions of direct light not intercepted by each canopy layer $N$ at time $K$ (sun inclination $I S$ ). This being so, it is readily seen that $X(K, N-1)$ is the fraction of leaves in layer $N$ receiving direct sunlight; for the fraction $X(K, N-1)$ of light not intercepted by layer $N-1$ must impinge upon the fraction $X(K, N-1)$ of the total area of layer $N$, and if it is assumed that the leaves are randomly distributed spatially throughout the planar extent of their respective layers, the fraction $X(K, N-1)$ of the leaves of layer $N$ must be illuminated, too. As a corollary to this reasoning, it is evident that those leaves in any layer $N$ receiving diffuse skylight only is

$$
D F(K, N)=1-X(K, N-1) .
$$

Furthermore, considering the distribution of direct light interception by the crop, we may write the ex- pression for the fractions of leaves in each layer which in addition to diffuse light also receive direct light within specified $\sin (L S)$ or $J$ intervals as

$$
D S(K, N, J)=F R(K, J) \times X(K, N-1) .
$$

All of these calculations are done by computer. To conserve space, however, these intermediate results will not be presented.

At this stage, the canopy has been divided into $N$ layers of $J$ radiation reception classes for each of $K$ solar altitudes. Since the theory beyond this point calls for actual radiation data, it is now necessary to compute the times at which the sun inclination of $5^{\circ}$, $15^{\circ}, \ldots, 85^{\circ}$ occur for the specific time and place in question. This information is readily obtained from the relation

$$
T(K)=\frac{1}{15} \arccos \left[\frac{\sin (I S)-\sin (L) \sin (D)}{\cos (L) \cos (D)}\right]
$$

where $T(K)$ is the time in hours before and after solar noon when a specitied altitude angle is obtained by the sun and $L$ and $D$ are, respectively, the latitude of the specific location and the declination of the sun for the specified date. Since the sun may not achieve an altitude angle of $85^{\circ}$ for all times and places, it is also necessary to solve the equation

$$
\sin (I S)=\cos (L) \cos (H) \cos (D)+\sin (L) \sin (D),
$$

when the hour angle $(H)$ equals zero, for the maximum value of $I S$.

Since light is both reflected and transmitted by the canopy leaves, we must calculate the intensities of light intercepted by each layer and thus available for this scattering. The first step is to calculate the incident diffuse skylight intercepted at each layer. It is based upon the same general principles used in the calculation of the penetration of direct sunlight. The sky is divided into zones of $10 \mathrm{deg}$ width, centered at inclinations of $5^{\circ}, 15^{\circ}, \ldots, 85^{\circ}$. Then, from Table III, the

Table III. The Relative Contribution to the Illuminance of a Horizontal Surface of 10 deg Zones from a Sky of Uniform Brightness ${ }^{a}$

$\begin{array}{llrrrrrrrrr}\text { Inclination in degrees } & 0 & 10 & 20 & 30 & 40 & 50 & 60 & 70 & 80 \\ \text { Relative contribution } & 0.030 & 0.087 & 0.133 & 0.163 & 0.174 & 0.163 & 0.133 & 0.087 & 0.030 & 90\end{array}$


Table IV. Diffuse Skylight and Direct Solar Radiation, in cal cm-2 $\mathrm{min}^{-1}$, as Reconstructed for 1 August 1961 at Ithaca, New York, for Sun Inclinations $I S=5^{\circ}, 15^{\circ}, \ldots, 65^{\circ}$

\begin{tabular}{lccccccc}
\hline$I S$ & 5 & 15 & 25 & 35 & 45 & 55 & 65 \\
\hline I)iffuse skylight & 0.000 & 0.027 & 0.056 & 0.082 & 0.109 & 0.122 & 0.150 \\
Direct solar radiation & 0.000 & 0.200 & 0.420 & 0.620 & 0.820 & 0.920 & 1.130 \\
\hline
\end{tabular}

penetration of diffuse light from these sections is determined as fractions of that incident upon a horizontal surface above the crop. These results are added together to give the penctration of diffuse light, from the entire sky. The solar times available from calculations of the previous paragraph then allow the proper intensities of diffuse skylight measured above the crop to be utilized in calculating the actual intensities of diffuse skylight incident on each canopy layer. By subtracting the results for each layer from those of the layer immediately preceding them, the diffuse skylight intercepted by each layer is then obtained; and multiplying these numbers by the sum of the reflectance and transmittance of the leaf material for diffuse skylight, the actual intensities of diffuse skylight scattered by each layer become available. A similar treatment of the intercepted direct sunlight added to these diffuse skylight results then gives the final distribution of the first set of scattered light sources in the canopy. Also, a source of scattered light is the underlying soil surface; and it is given a strength equivalent to twice its reflectance.

If we now represent the strength of the scattered light source of a layer $N$ by $S T(N)$, and if we let $X(N)$ represent the penetration of diffuse light through $N$ successive layers (calculated in the previous paragraph), we can rapidly complete the theory. First, since the transmitted and reflected light are prictically ideally scattered, ${ }^{12}$ approximately half of the light scattered at a layer goes up and the other half down, thus making it possible to express the contribution of layer 1 to the illuminance of layer $N$ as $0.5 S T(1) Z(N-1)$. Similarly, the contribution from layer 2 may be written as $0.5 S T(2) Z(N-2)$, the contribution from layer $N-1$ as $0.5 S T(N-1) Z(1)$, the contribution from layer $N$ +1 as $0.5 S T(N+1) Z(1)$, the contribution from the last layer, $N M A X$, as $0.5 S T(N M A X) Z(N M A X-N)$, and the contribution from layer $N M A X+1$, the soil surface, as $0.5 S T(N M / A X+1) Z(N M A X+1-N)$. (The use of the factor 0.5 here is what necessitated multiplying the reflectance of the soil by two in the previous paragraph to obtain its scattering strength.)

Writing these expressions in summation form, we get two separate relations:

$$
\begin{gathered}
D(N)=0.5 \sum_{L=1}^{N-1} S \eta^{\prime}(L) \times Z(N-L), \\
U(N)=0.5 \sum_{L=N+1}^{N M A X+1} S T(L) \times Z(L-N) .
\end{gathered}
$$

$D(N)$ represents the intensity of scattered light incident on layer $N$ from all lajers above it; and $U(N)$ repre- layers below it. The amounts of these fluxes that are intercepted by each layer may also be calculated and secondary scattered light sources created. Two or three such cycles are sufficient to account for essentially all scattered light. Thus, at the end, one has available the profile of light moving upward through the canopy, $U(N)$, and by adding $D(N)$ to the penetration of direct sunlight and diffuse skylight, the profile of light moving downward through the canopy. The upper limit of $U(N)$ divided by incident solar radiation and skylight gives crop reflectance; and a similar operation upon the lower limit of transmitted light gives crop transmittance.

\section{Application}

In applying D-I theory, three of the most important pieces of data required are the scattering coefficients of the individual plant leaf material for direct solar radiation, diffuse skylight, and light transmitted through vegetation. From spectral measurements of reflectance and transmittance made of a corn leaf by Yocum et al. ${ }^{13}$ and spectral distributions of these three energy fluxes published by Gates, ${ }^{14}$ these factors calculated for corn are $0.54,0.21$, and 0.62 , respectively. The first two of these three coefficients are used in computing the strengths of the original scattering sources, and the third is used in computing the strengths of succeeding scattering sources.

Also needed is the leaf distribution function of the crop; and Fig. 1 includes this function for corn as measured by de Wit. ${ }^{6}$ Then, leaf area index and

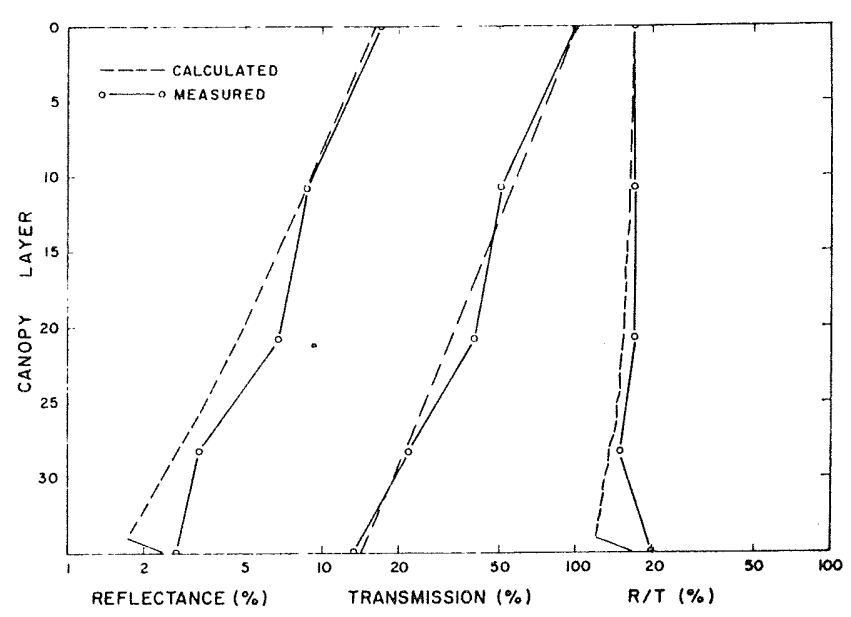

Fig. 2. Calculated and measured profiles of reflection $R$ and transmission $T$ in a corn crop. $R$ is computed as upward moving radiation divided by incident radiation-and $T$ as dowaward mov- 


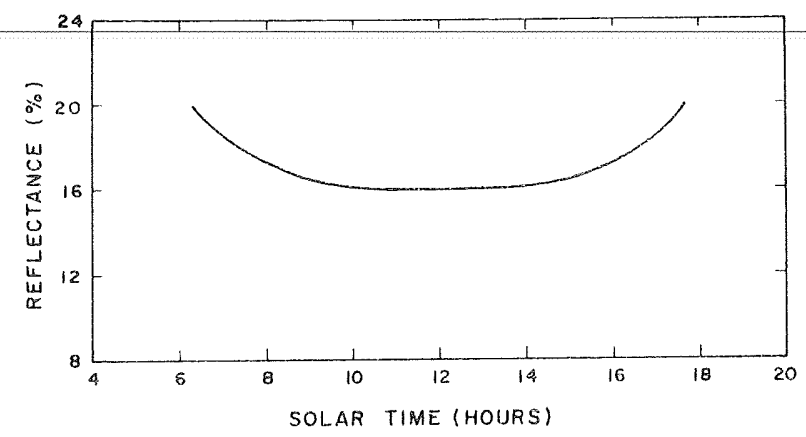

Fig. 3. Calculated reflectance of a corn crop as a function of time for clear day conditions.

canopy density are required. We have determined 0.1 to be a good value of canopy density for corn; and leaf area index is determined for the specific stage of growth by measurement. The application of D-I theory presented will be for a corn crop growing at Ithaca, New York, on 1 August 1961. The leaf area index measured for that situation by Baker and Musgrave $^{15}$ was 3.5 . This value, together with the canopy density of 0.1 , yields thirty-five crop layers for investigation.

Finally, direct solar radiation and diffuse skylight data are needed. Solving Eq. (9) it is found that sun elevations of $75^{\circ}$ and $85^{\circ}$ are not reached at Ithaca on 1 August; so they are omitted from further consideration. Then, solving Eq. (8) yields values of 6.7, 5.7, $4.8,3.9,3.0,2.0$, and 0.5 for the hours before and after solar noon when the sun occupies altitudinal positions of $5^{\circ}, 15^{\circ}, \ldots, 65^{\circ}$, respectively. At these times, data from Lemon and Wright, ${ }^{16}$ Allen et al. ${ }^{13}$ and Threlkeld ${ }^{17}$ yield values of direct and diffuse radiation fluxes above the crop as contained in Table IV.

The results of using these data in the manner prescribed in Sec. II are shown in Figs. 2 and 3 . Also included in Fig. 2 are some experimental measurements made by Allen and Brown ${ }^{18}$ on this crop six weeks later and Allen et al. ${ }^{19}$ about the same time. The three pair of values for reflection and transmission within the crop and the one at the bottom of the crop are the work of the former authors; the pair at the top of the crop are the work of the latter group. Originally, Allen and Brown had reported reflection above the crop, too. However, their value was not measured but estimated from certain assumptions. The value of Allen et al. ${ }^{19}$ was the mean of measurements carried out on several days around 10 September 1961.

\section{Discussion}

The degree of correspondence between the measured and calculated profiles of reflection and transmission in Fig. 2 is truly remarkable, especially considering the fact that upper and lower limits of these two parameters were not matched, as was done in the evaluation of $\mathrm{K}-\mathrm{M}$ theory by Allen and Brown. ${ }^{18}$ Considering problems associated with canopy radiation measurements of this type, the verifieation of D I theory must be considered virtually complete, particularly when the reflection coefficient for the crop in Fig. 3 is integrated over the day to give the same average value as measured by Allen et al. ${ }^{19}$

Besides being more versatile than $K-M$ theory in predicting changes in canopy reflection and transmission with solar altitude, D-I theory has another advantage quite apart from remote sensing; and this is that it is particularly well suited for inclusion in computer simulation models of canopy photosynthesis. From K-M theory, for instance, all a person can glean about the light reception at various canopy layers is its average intensity. Monteith ${ }^{20}$ has demonstrated quite vividly, however, that photosynthesis calculated from a mean light intensity spread over all the leaf area of a given layer can be much different from that calculated from the same amount of radiation concentrated in sunflecks of a correspondingly smaller area. D-I theory even provides more information than this, giving the fraction of leaves in each layer receiving diffuse light only and the fractions which also receive direct light at various angles of inclination to the rays of the sun. Thus, D-I theory improves the interpretative capabilities of remote sensing and provides a sound theoretical framework for the photosynthetic modeling of plant canopies.

This is a joint contribution from the Soil and Water Conservation Research Division, Agricultural Research Service, U.S. Department of Agriculture, the Agricultural University, Wageningen, and the Institute for Biological and Chemical Research, Wageningen, The Netherlands.

\section{Appendix A}

The purpose of this appendix is to present a detailed derivation of the light distribution function from relations in evidence in Fig. 4. Part $A$ of Fig. 4 represents a plant leaf of given orientation with respect to the soil surface, and the line TS a ray of the sun. Part $B$ is the same configuration seen from a different point of view. The angles $I S, I L, D A$, and $L S$ are, respectively, the inclination of the sun, the inclination of the leaf, the difference between the azimuths of the leaf and the sun, and the angle between the leaf and the rays of the sun.

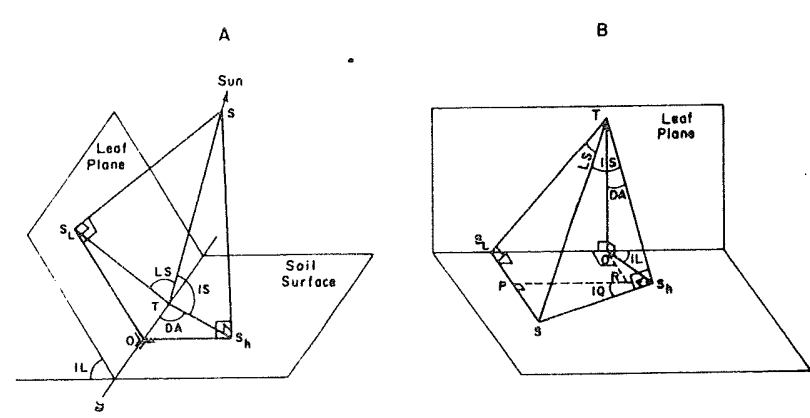

Fig. 4. Geometrical representations of the relations between \& plant leaf, the soil surface, and a ray of the sun. 


$$
\begin{gathered}
T S_{h}=T S \times \cos (I S), \\
O S_{h}=T S_{h} \times \sin (D A),
\end{gathered}
$$

and

$$
O R=O S_{h} \times \sin (I L)
$$

thus making $O R$ also equivalent to

$$
O R=T S \times[\cos (I S) \sin (I L) \sin (D A)] .
$$

Next, it can be seen that

$$
S S_{h}=T S \times \sin (I S)
$$

and that

$$
P S=S S_{h} \times \sin (I Q)
$$

thus making

$$
P S=T S \times[\sin (I S) \sin (I Q)] .
$$

However, since it is evident that $\sin (I Q)=\cos (I L)$,

$$
P S=T S \times[\sin (I S) \cos (I L)] .
$$

Then, since $O R=S_{L} P$ and $S S_{L}=S_{L} P+P S$, we have

$S S_{L}=T S \times[\sin (I S) \cos (I L)+\cos (I S) \sin (I L) \sin (D A)]$.

A second way of expressing $S S_{L}$, which is evident from Fig. 4, is

$$
S S_{L}=T S \times \sin (L S) .
$$

Combining this equation with the previous one for $S S_{L}$ and solving for $\sin (L S)$ then yields

$$
\sin (L S)=A+B \times \sin (D A),
$$

where

$$
\begin{aligned}
A & =\sin (I S) \cos (I L), \\
B & =\cos (I S) \sin (I L) .
\end{aligned}
$$

Now the light distribution function $S(J)$ for a given value of $I S$ and $I L$ is defined as the probability that a light ray is intercepted by a leaf with a sine of its angle to the light equal to or smaller than $\sin (L S)$. Since the amount of light intercepted by the leaves in a small azimuth interval is proportional to the size $d(D D A)$ of this interval and since this amount of intercepted light is also proportional to the projection of the leaf surface elements in the direction of the sun, that is with $\sin (L S)$, the light distribution function $S(J)$, for $J=\sin (L S)$, is

$$
\begin{aligned}
S(J)=\left[-\int_{-\pi / 2}^{D A O}\right. & \sin (L S) \times d(D D A) \\
& \left.+\int_{D A O}^{D A} \sin (L S) \times d(D D A)\right] \times W,
\end{aligned}
$$

where $W$ is a proportionality constant.

The reason for doing this integration in two parts is to distinguish between light falling on the uppersides and on the undersides of the leaves. Since light is parallel to a leaf when $\sin (L S)=0$, the boundary angle for $I S<I L$. Since no light falls on the undersides of the leaves if $I S \geq I L$, the boundary angle in this case is defined as $-\pi / 2$.

To determine the value of $W$, we note that if the integration is carried out to the maximum value of $D A$, that is $\pi / 2$, we must have $S(J)=1$, or

$$
\begin{aligned}
1=\left[-\int_{-\pi / 2}^{D A O}\right. & \sin (L S) \times d(D D A) \\
& \left.+\int_{D A O}^{\pi / 2} \sin (L S) \times d(D D A)\right] \times W .
\end{aligned}
$$

Evaluating the first of these integrals, after substituting for $\sin (L S)$ the expression in Eq. (A11), we have

$$
\begin{array}{r}
-\int_{-\pi / 2}^{D A O} \sin (L S) \times d(D D A)=-A \times[D A O+(\pi / 2)] \\
+B \times \cos (D A O)
\end{array}
$$

and evaluating the second integral in a similar fashion yields

$$
\begin{array}{r}
\left.\int_{D A O}^{\pi / 2} \sin (L S) \times d(D D A)=A \times[(\pi / 2)-D A O)\right] \\
+B \times \cos (D A O) .
\end{array}
$$

Our initial expression (A16) thus reduces to

$1=[-2 \times A \times D A O+2 \times B \times \cos (D A O)] \times W$,

from which the proportionality constant $W$ is determined to be

$$
W=[2 \times B \times \cos (D A O)-2 \times A \times D A O]^{-1} .
$$

Now, for $D A<D A O$, the light distribution function $S(J)$ will be given by the integral

$S(J)=\left\{-\int_{-\pi / 2}^{D A}[A+B \times \sin (D D A)] \times d(D D A)\right\} \times W$.

Evaluating this expression,

$S(J)=\{B \times \cos (D A)-A \times[(\pi / 2)+(D A)]\} \times W . \quad$ (A22)

For $D A \geq D A O$, the light distribution function must be evaluated from the sum of the two integrals

$$
\begin{aligned}
S(J) & =\left\{-\int_{-\pi / 2}^{D A O}[A+B \times \sin (D D A)] \times d(D D A)\right. \\
+ & \left.\int_{D A O}^{D A}[A+B \times \sin (D D A)] \times d(D D A)\right\} \times W .
\end{aligned}
$$

The first of these integrals results in the same expression as was obtained for the previous integral, except now $D A$ is replaced by $D A O$. The second integral, $S J 2$, results in the expression

$$
\begin{aligned}
S J 2=[A \times D A-A \times D A O & -B \times \cos (D A) \\
& +B \times \cos (D A O)] \times W .
\end{aligned}
$$

Combining these results together, we get, for the light distribution function for $D A \geq D A O$,

$S(J)=\{B \times[2 \times \cos (D A O)-\cos (D A)]-A$ 
Appendix B. This Appendix Contains the Eighty-One Light Distribution Functions for All Combinatlons of the Nine Sut Inclinatlons $\left(I S=5^{\circ}, 15^{\circ}, \ldots, 85^{\circ}\right)$ and the Nive Larif Inclinations $\left(I L=5^{\circ}, 15^{\circ}, \ldots, 85^{\circ}\right)$

\begin{tabular}{|c|c|c|c|c|c|c|c|c|c|c|c|}
\hline \multirow[b]{2}{*}{$I S$} & \multirow[b]{2}{*}{$I L$} & \multirow[b]{2}{*}{0.1} & \multirow[b]{2}{*}{0.2} & \multirow[b]{2}{*}{0.3} & \multicolumn{4}{|c|}{$S(J)$ for $\sin (L S) \leq$} & \multirow[b]{2}{*}{0.8} & \multirow[b]{2}{*}{0.9} & \multirow[b]{2}{*}{1.0} \\
\hline & & & & & 0.4 & 0.5 & 0.6 & 0.7 & & & \\
\hline 5 & 5 & 0.234 & 1.000 & 1.000 & 1.000 & 1.000 & 1.000 & 1.000 & 1.000 & 1.000 & 1.000 \\
\hline 5 & 15 & 0.081 & 0.405 & 0.651 & 1.000 & 1.000 & 1.000 & 1.000 & 1.000 & 1.000 & 1.000 \\
\hline 5 & 25 & 0.029 & 0.122 & 0.316 & 0.617 & 0.999 & 1.000 & 1.000 & 1.000 & 1.000 & 1.000 \\
\hline 5 & 35 & 0.015 & 0.063 & 0.150 & 0.292 & 0.626 & 0.787 & 1.000 & 1.000 & 1.000 & 1.000 \\
\hline 5 & 45 & 0.010 & 0.041 & 0.095 & 0.178 & 0.299 & 0.493 & 0.770 & 1.000 & 1.000 & 1.000 \\
\hline 5 & 55 & 0.008 & 0.031 & 0.070 & 0.129 & 0.210 & 0.324 & 0.494 & 0.791 & 1.000 & 1.000 \\
\hline 5 & 65 & 0.006 & 0.025 & 0.157 & 0.104 & 0.167 & 0.253 & 0.370 & 0.542 & 0.847 & 1.000 \\
\hline 5 & 75 & 0.005 & 0.022 & 0.050 & 0.091 & 0.146 & 0.218 & 0.314 & 0.445 & 0.651 & 1.000 \\
\hline 5 & 85 & 0.005 & 0.021 & 0.047 & 0.085 & 0.136 & 0.203 & 0.291 & 0.408 & 0.579 & 1.000 \\
\hline 15 & 5 & 0.000 & 0.183 & 0.577 & 1.000 & 1.000 & 1.000 & 1.000 & 1.000 & 1.000 & 1.000 \\
\hline 15 & 15 & 0.041 & 0.124 & 0.252 & 0.450 & 0.998 & 1.000 & 1.000 & 1.000 & 1.000 & 1.000 \\
\hline 15 & 25 & 0.033 & 0.168 & 0.232 & 0.326 & 0.463 & 0.696 & 1.000 & 1.000 & 1.000 & 1.000 \\
\hline 15 & 35 & 0.017 & 0.070 & 0.181 & 0.344 & 0.420 & 0.526 & 0.692 & 1.000 & 1.000 & 1.000 \\
\hline 15 & 45 & 0.011 & 0.044 & 0.104 & 0.201 & 0.431 & 0.500 & 0.593 & 0.736 & 1.000 & 1.000 \\
\hline 15 & 55 & 0.008 & 0.033 & 0.075 & 0.139 & 0.232 & 0.379 & 0.574 & 0.666 & 0.817 & 1.000 \\
\hline 15 & 65 & 0.007 & 0.026 & 0.061 & 0.111 & 0.181 & 0.277 & 0.418 & 0.654 & 0.759 & 1.000 \\
\hline 15 & 75 & 0.006 & 0.023 & 0.053 & 0.097 & 0.156 & 0.235 & 0.342 & 0.497 & 0.759 & 1.000 \\
\hline 15 & 85 & 0.005 & 0.022 & 0.050 & 0.091 & 0.146 & 0.218 & 0.314 & 0.445 & 0.651 & 1.000 \\
\hline 25 & 5 & 0.000 & 0.000 & 0.000 & 0.357 & 0.998 & 1.000 & 1.000 & 1.000 & 1.000 & 1.000 \\
\hline 25 & 15 & 0.000 & 0.068 & 0.185 & 0.306 & 0.460 & 0.699 & 1.000 & 1.000 & 1.000 & 1.000 \\
\hline 25 & 25 & 0.021 & 0.062 & 0.120 & 0.196 & 0.296 & 0.429 & 0.632 & 1.000 & 1.000 & 1.000 \\
\hline 25 & 35 & 0.022 & 0.108 & 0.147 & 0.199 & 0.269 & 0.359 & 0.480 & 0.664 & 1.000 & 1.000 \\
\hline 25 & 45 & 0.013 & 0.053 & 0.137 & 0.259 & 0.310 & 0.376 & 0.461 & 0.578 & 0.770 & 1.000 \\
\hline 25 & 55 & 0.009 & 0.038 & 0.088 & 0.170 & 0.366 & 0.419 & 0.486 & 0.575 & 0.705 & 1.000 \\
\hline 25 & 65 & 0.007 & 0.030 & 0.070 & 0.129 & 0.215 & 0.350 & 0.529 & 0.605 & 0.713 & 1.000 \\
\hline 25 & 75 & 0.007 & 0.026 & 0.061 & 0.111 & 0.181 & 0.277 & 0.418 & 0.654 & 0.759 & 1.000 \\
\hline 25 & 85 & 0.006 & 0.025 & 0.057 & 0.104 & 0.167 & 0.253 & 0.370 & 0.542 & 0.847 & 1.000 \\
\hline 35 & 5 & 0.000 & 0.000 & 0.000 & 0.000 & 0.000 & 0.595 & 1.000 & 1.000 & 1.000 & 1.000 \\
\hline 35 & 15 & 0.000 & 0.000 & 0.000 & 0.157 & 0.300 & 0.451 & 0.653 & 1.000 & 1.000 & 1.000 \\
\hline 35 & 25 & 0.000 & 0.044 & 0.117 & 0.189 & 0.270 & 0.368 & 0.493 & 0.676 & 1.000 & 1.000 \\
\hline 35 & 35 & 0.015 & 0.045 & 0.085 & 0.138 & 0.203 & 0.283 & 0.385 & 0.522 & 0.740 & 1.000 \\
\hline 35 & 45 & 0.018 & 0.090 & 0.120 & 0.162 & 0.216 & 0.283 & 0.368 & 0.480 & 0.640 & 1.000 \\
\hline 35 & 55 & 0.012 & 0.049 & 0.125 & 0.237 & 0.283 & 0.340 & 0.414 & 0.510 & 0.645 & 1.000 \\
\hline 35 & 65 & 0.009 & 0.038 & 0.088 & 0.170 & 0.366 & 0.419 & 0.486 & 0.575 & 0.705 & 1.000 \\
\hline 35 & 75 & 0.008 & 0.033 & 0.075 & 0.139 & 0.232 & 0.379 & 0.574 & 0.666 & 0.817 & 1.000 \\
\hline 35 & 85 & 0.008 & 0.031 & 0.070 & 0.129 & 0.210 & 0.324 & 0.494 & 0.791 & 1.000 & 1.000 \\
\hline 45 & 5 & 0.000 & 0.000 & 0.000 & 0.000 & 0.000 & 0.000 & 0.449 & 1.000 & 1.000 & 1.000 \\
\hline 45 & 15 & 0.000 & 0.000 & 0.000 & 0.000 & 0.000 & 0.274 & 0.445 & 0.655 & 1.000 & 1.000 \\
\hline 45 & 25 & 0.000 & 0.000 & 0.000 & 0.114 & 0.213 & 0.309 & 0.418 & 0.553 & 0.760 & 1.000 \\
\hline 45 & 35 & 0.000 & 0.036 & 0.097 & 0.154 & 0.219 & 0.294 & 0.383 & 0.496 & 1.654 & 1.000 \\
\hline 45 & 45 & 0.014 & 0.041 & 0.077 & 0.124 & 0.182 & 0.252 & 0.339 & 0.450 & 0.604 & 1.000 \\
\hline 45 & 55 & 0.018 & 0.090 & 0.120 & 0.162 & 0.216 & 0.283 & 0.368 & 0.480 & 0.640 & 1.000 \\
\hline 45 & 65 & 0.013 & 0.053 & 0.137 & 0.259 & 0.310 & 0.376 & 0.461 & 0.578 & 0.770 & 1.000 \\
\hline 45 & 75 & 0.011 & 0.044 & 0.104 & 0.201 & 0.431 & 0.500 & 0.593 & 0.736 & 1.000 & 1.000 \\
\hline 45 & 85 & 0.010 & 0.041 & 0.095 & 0.178 & 0.299 & 0.493 & 0.770 & 1.000 & 1.000 & 1.000 \\
\hline 55 & 5 & 0.000 & 0.000 & 0.000 & 0.000 & 0.000 & 0.000 & 0.000 & 0.378 & 1.000 & 1.000 \\
\hline 55 & 15 & 0.000 & 0.000 & 0.000 & 0.000 & 0.000 & 0.000 & 0.242 & 0.459 & 0.721 & 1.000 \\
\hline 55 & 25 & 0.000 & 0.000 & 0.000 & 0.000 & 0.000 & 0.216 & $0.342^{\circ}$ & 0.475 & 0.646 & 1.000 \\
\hline 55 & 35 & 0.000 & 0.000 & 0.000 & 0.103 & 0.193 & 0.278 & 0.373 & 0.485 & 0.633 & 1.000 \\
\hline 55 & 45 & 0.000 & 0.036 & 0.097 & 0.154 & 0.219 & 0.294 & 0.383 & 0.496 & 0.654 & 1.000 \\
\hline 55 & 55 & 0.015 & 0.045 & 0.085 & 0.138 & 0.203 & 0.283 & 0.385 & 0.522 & 0.740 & 1.000 \\
\hline 55 & 65 & 0.022 & 0.108 & 0.147 & 0.199 & 0.269 & 0.359 & 0.480 & 0.664 & 1.000 & 1.000 \\
\hline 55 & 75 & 0.017 & 0.070 & 0.181 & 0.344 & 0.420 & 0.526 & 0.692 & 1.000 & 1.000 & 1.000 \\
\hline 55 & 85 & 0.015 & 0.063 & 0.150 & 0.292 & 0.626 & 0.788 & 1.000 & 1.000 & 1.000 & 1.000 \\
\hline 65 & 5 & 0.000 & 0.000 & 0.000 & 0.000 & 0.000 & 0.000 & 0.000 & 0.000 & 0.462 & 1.000 \\
\hline 65 & 15 & 0.000 & 0.000 & 0.000 & 0.000 & 0.000 & 0.000 & 0.000 & 0.229 & 0.533 & 1.000 \\
\hline 65 & 25 & 0.000 & 0.000 & 0.000 & 0.000 & 0.000 & 0.000 & 0.211 & 0.393 & 0.583 & 1.000 \\
\hline 65 & 35 & 0.000 & 0.000 & 0.000 & 0.000 & 0.000 & 0.216 & 0.342 & 0.475 & 0.646 & 1.000 \\
\hline
\end{tabular}


Appendix B. (continued)

\begin{tabular}{|c|c|c|c|c|c|c|c|c|c|c|c|}
\hline \multirow[b]{2}{*}{$I S$} & \multirow[b]{2}{*}{$I L$} & \multicolumn{10}{|c|}{$S(J)$ for $\sin (L S) \leq$} \\
\hline & & 0.1 & 0.2 & 0.3 & 0.4 & 0.5 & 0.6 & 0.7 & 0.8 & 0.9 & 1.0 \\
\hline 65 & 45 & 0.000 & 0.000 & 0.000 & 0.114 & 0.213 & 0.309 & 0.418 & 0.553 & 0.760 & 1.000 \\
\hline 65 & 55 & 0.000 & 0.044 & 0.117 & 0.189 & 0.270 & 0.368 & 0.493 & 0.676 & 1.000 & 1.000 \\
\hline 65 & 65 & 0.021 & 0.062 & 0.120 & 0.196 & 0.296 & 0.429 & 0.632 & 1.000 & 1.000 & 1.000 \\
\hline 65 & 75 & 0.033 & 0.168 & 0.232 & 0.326 & 0.464 & 0.696 & 1.000 & 1.000 & 1.000 & 1.000 \\
\hline 65 & 85 & 0.029 & 0.122 & 0.316 & 0.617 & 1.000 & 1.000 & 1.000 & 1.000 & 1.000 & 1.000 \\
\hline 75 & 5 & 0.000 & 0.000 & 0.000 & 0.000 & 0.000 & 0.000 & 0.000 & 0.000 & 0.000 & 1.000 \\
\hline 75 & 15 & 0.000 & 0.000 & 0.000 & 0.000 & 0.000 & 0.000 & 0.000 & 0.000 & 0.316 & 1.000 \\
\hline 75 & 25 & 0.000 & 0.000 & 0.000 & 0.000 & 0.000 & 0.000 & 0.000 & 0.229 & 0.533 & 1.000 \\
\hline 75 & 35 & 0.000 & 0.000 & 0.000 & 0.000 & 0.000 & 0.000 & 0.242 & 0.459 & 0.721 & 1.000 \\
\hline 75 & 45 & 0.000 & 0.000 & 0.000 & 0.000 & 0.000 & 0.274 & 0.445 & 0.655 & 1.000 & 1.000 \\
\hline 75 & 55 & 0.000 & 0.000 & 0.000 & 0.157 & 0.300 & 0.451 & 0.653 & 1.000 & 1.000 & 1.000 \\
\hline 75 & 65 & 0.000 & 0.068 & 0.185 & 0.306 & 0.460 & 0.699 & 1.000 & 1.000 & 1.000 & 1.000 \\
\hline 75 & 85 & 0.081 & 0.405 & 0.651 & 1.000 & 1.000 & 1.000 & 1.000 & 1.000 & 1.000 & 1.000 \\
\hline 85 & 5 & 0.000 & 0.000 & 0.000 & 0.000 & 0.000 & 0.000 & 0.000 & 0.000 & 0.000 & 1.000 \\
\hline 85 & 15 & 0.000 & 0.000 & 0.000 & 0.000 & 0.000 & 0.000 & 0.000 & 0.000 & 0.000 & 1.000 \\
\hline 85 & 25 & 0.000 & 0.000 & 0.000 & 0.000 & 0.000 & 0.000 & 0.000 & 0.000 & 0.462 & 1.000 \\
\hline 85 & 35 & 0.000 & 0.000 & 0.000 & 0.000 & 0.000 & 0.000 & 0.000 & 0.378 & 1.000 & 1.000 \\
\hline 85 & 45 & 0.000 & 0.000 & 0.000 & 0.000 & 0.000 & 0.000 & 0.449 & 1.000 & 1.000 & 1.000 \\
\hline 85 & 55 & 0.000 & 0.000 & 0.000 & 0.000 & 0.000 & 0.595 & 1.000 & 1.000 & 1.000 & 1.000 \\
\hline 85 & 65 & 0.000 & 0.000 & 0.000 & 0.357 & 1.000 & 1.000 & 1.000 & 1.000 & 1.000 & 1.000 \\
\hline 85 & 75 & 0.000 & 0.183 & 0.577 & 1.000 & 1.000 & 1.000 & 1.000 & 1.000 & 1.000 & 1.000 \\
\hline 85 & 85 & 0.234 & 1.000 & 1.000 & 1.000 & 1.000 & 1.000 & 1.000 & 1.000 & 1.000 & 1.000 \\
\hline
\end{tabular}

\section{References}

1. A. C. Handy and F. H. Perrin, The Principles of Optics (McGraw-Hill Book Co., New York, 1932), Chap. 1, p. 24.

2. V. I. Myers and W. A. Allen, Appl. Opt. 7, 1819 (1968).

3. P. Kubelka and F. Munk, Z. Tech. Physik 11, 593 (1931).

4. W. A. Allen and A. J. Richardson, J. Opt. Soc. Amer. 58, 1023 (1968).

5. A. A. Nichiporovich, Soviet Plant Physiol. 8, 428 (1961).

6. C. T. de Wit, "Photosynthesis of Leaf Canopies," Agr. Res. Rept. 663 (Wageningen, 1965), Chap. 4, p. 11

7. L. J. Fritschen, Agr. Meteorol. 4, 55 (1967).

8. Lin-Sien Chia, Quart. J. Roy. Meteorol. Soc. 93, 116 (1967).

9. J. L. Monteith and G. Szeicz, Quart. J. Roy. Meteorol. Soc. 87, 159 (1961).

10. S. B. Idso, D. G. Baker, and B. L. Blad, Quart. J. Roy. Meteorol. Soc. 95, 244 (1969).
11. M. Monsi and T. Saeki, Japan. J. Botany 14, 22 (1953).

12. E. I. Rabinowitch, Photosynthesis and Related Pracesses (Interscience Publishers, New York, 1951), Vol. 2, Part 1.

13. C. S. Yocum, L. H. Allen, and E. R. Lemon, Agron. J. 56, 249 (1964).

14. D. M. Gates, in Agricultural Meteorology (American Meteorological Society, Boston, 1965), p. 1.

15. D. N. Baker and R. B. Musgrave, Crop Sci. 4, 127 (1964).

16. J. L. Wright and E. R. Lemon, Agron. J. 58, 265 (1966).

17. J. L. Threlkeld, Thermal Environmental Engineering (Prentice-Hall, Englewood Cliffs, N.J., 1962), Chap. 14, p. 329.

18. L. H. Allen and K. W. Brown, Agron. J. 57, 575 (1965).

19. L. H. Allen, C. S. Yocum, and E. R. Lemon, Agron. J. 56, 253 (1964).

20. J. L. Monteith, Ann. Botany (London) 29, 17 (1965). 